\title{
PUBLICACIONES DE LA REVISTA AGRONOMÍA MESOAMERICANA DURANTE EL PERIODO 1990-2010: CLASIFICACIÓN, TEMÁTICA, PAÍSES Y DIFUSIÓN ${ }^{1}$
}

\author{
Nelson Peña Navarro ${ }^{2}$
}

\begin{abstract}
RESUMEN
Publicaciones de la revista Agronomía Mesoamericana durante el periodo 1990-2010: Clasificación, temática, países y difusión. El objetivo de este trabajo fue determinar la temática y procedencia de las publicaciones de la revista Agronomía Mesoamericana durante el periodo comprendido entre 1990-2010. Esta revista es el Órgano divulgativo del Programa Cooperativo Centroamericano para el Mejoramiento de Cultivos y Animales (PCCMCA). Desde el inicio en 1990 a la fecha se han publicado 629 trabajos de los cuales el $63 \%$ son artículos científicos, $26 \%$ notas técnicas, $8 \%$ son análisis y comentarios, mientras que las informaciones técnicas y las revisiones bibliográficas poseen $2 \%$ cada uno. Costa Rica y México son los países con mayor cantidad de trabajos publicados, con 37 y $32 \%$ respectivamente, seguidos de El Salvador, Guatemala, Honduras y Cuba. Los principales temas tratados son el maíz (24\%) y el frijol (21\%), seguidos de los forrajes $(7 \%)$ y el de ganado lechero $(4 \%)$, mientras que el arroz, el sorgo, las malezas y el pejibaye alcanzaron el $3 \%$ cada uno. El porcentaje de rechazo de los trabajos sometidos a publicación durante el 2010 fue del $20 \%$. Se destaca la distribución impresa de la revista a treinta países alrededor del mundo, a bibliotecas así como a centros de documentación principalmente a los continentes de América, Europa, Asia y algunas de las islas del Caribe.
\end{abstract}

Palabras claves: PCCMCA, revista agropecuaria, publicación, países mesoamericanos, investigaciones científicas.

\begin{abstract}
Publications of the Mesoamerican Journal of Agronomy during the period 1990-2010: Classification, thematic, countries and diffusion. The objective of this study was to assess the thematic and sources of the publications in the Mesoamerican Journal of Agronomy during the period 1990-2010. Mesoamerican Journal of Agronomy was created as a publication of the Central American Cooperative Program for the Improvement of Crops and Livestock (PCCMCA). From 1990 to date, 629 papers have been published, of which $63 \%$ are scientific articles, $26 \%$ are technical notes, $8 \%$ are analyses and commentaries, while technical information and literature reviews are $2 \%$ each. Costa Rica and Mexico are the countries with the largest number of published papers, with 37 and $32 \%$ respectively, followed by El Salvador, Guatemala, Honduras and Cuba. The principal topics have been corn $(24 \%)$ and beans $(21 \%)$, forages $(7 \%)$, and dairy cattle $(4 \%)$, while rice, sorghum, weeds and peach palm reached $3 \%$ each. In 2010, the journal rejected $20 \%$ of all papers submitted for publication. We highlight the distribution of the printed journal to libraries and documentation centers in thirty countries around the world, mainly the continents of America, Europa, Asia and some Caribbean islands.
\end{abstract}

Key words: PCCMCA, agricultural journal, publication, Mesoamerican countries, scientific research.

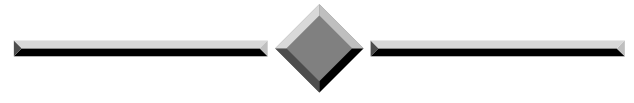

1 Recibido: 24 de febrero, 2011. Aceptado: 16 de mayo, 2011.

2 Revista Agronomía Mesoamericana. Estación Experimental Fabio Baudrit Moreno, Universidad de Costa Rica. Alajuela, Costa Rica. nelson7685@yahoo.es 


\section{INTRODUCCIÓN}

La revista Agronomía Mesoamericana fue creada como Órgano divulgativo del Programa Cooperativo Centroamericano para el Mejoramiento de Cultivos y Animales (PCCMCA) en 1990.

El PCCMCA tuvo como punto de partida el Proyecto Centroamericano de Mejoramiento de Maíz (PCCMM) en 1954, el cual estuvo dirigido por el Dr. Edwin J. Wellhausen, su principal objetivo fue establecer un programa cooperativo que analizara la situación en que se encontraba el mejoramiento del maíz de esa época (Fuentes et al. 1990). Luego del éxito que tuvo el PCCMM y de la inclusión de otros cultivos como el arroz, frijol, sorgo e investigaciones pecuarias se cambia las siglas por PCCMCA (Wellhausen 1990); en esta se busca principalmente el intercambio de ideas y conocimientos técnico-científicos entre los profesionales e instituciones de la región mesoamericana (Morera y Astorga 1993).

La revista Agronomía Mesoamericana remonta sus orígenes a 1989 cuando se realiza en Honduras la XXXV reunión anual del PCCMCA y debido a la necesidad de difundir los trabajos expuestos por los principales investigadores de la región, la cual en su momento comprendía los países de Centroamérica y El Caribe (Wellhausen 1990). La primera publicación de la revista se realiza en 1990 en un volumen y presentado en la XXXVI reunión del PCCMCA efectuada en El Salvador. Este evento tuvo como tema principal el establecimiento de una red mesoamericana de recursos fitogenéticos (Morera y Astorga 1993).

En el primer volumen se publicaron ocho artículos y la misma cantidad de notas técnicas, además de dos trabajos considerados como análisis y comentarios, el editor principal en esa época era el Ing. Juan Manuel Herrera Contreras de Guatemala. En los primeros cinco años de publicación, la revista imprimió un solo volumen anual y a partir de 1996 dos números semestrales, a excepción del 2004 y el 2006, donde se publicó un número extraordinario. El primero sobre consumo de frijol y la nutrición humana, y el segundo sobre fitomejoramiento participativo. Actualmente la revista Agronomía Mesoamericana tiene 21 años de publicarse ininterrumpidamente.

El objetivo del presente trabajo fue determinar la temática y procedencia de las publicaciones de la revista Agronomía Mesoamericana durante el periodo comprendido entre 1990-2010.

\section{Clasificación de la revista}

La revista clasifica todos los trabajos principalmente en cinco secciones: Artículo, Nota Técnica, Análisis y Comentarios, Información técnica y Revisión Bibliográfica.

Todos los trabajos presentados, para ser aceptados en el proceso de edición deberán cumplir con todos los requisitos de fondo y formato de la revista Agronomía Mesoamericana.

La revista ha publicado 629 trabajos de los cuales 394 son artículos científicos (63\%) (Figura 1).

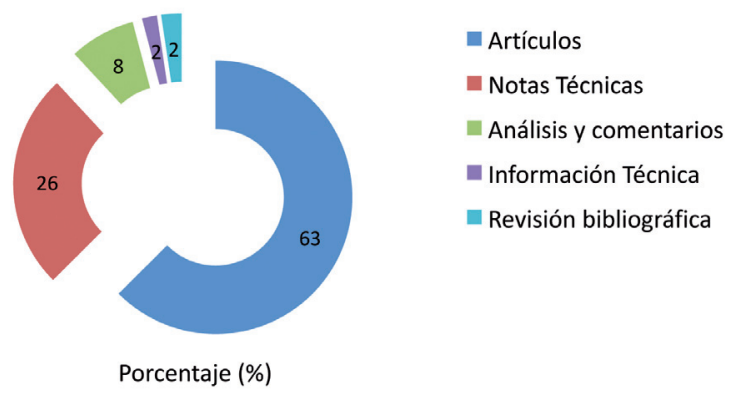

Figura 1. Porcentaje de trabajos publicados durante el periodo 1990-2010 de acuerdo con la clasificación otorgada por la revista Agronomía Mesoamericana. Costa Rica, 2011.

Las Notas Técnicas ocupan el segundo lugar en publicaciones alcanzando un $26 \%$, seguida de los Análisis y Comentarios con el 8\%, y porcentualmente las Informaciones Técnicas junto a las Revisiones Bibliográficas representan la misma cantidad (2\%).

\section{Países que publican}

En la revista Agronomía Mesoamericana se publican investigaciones provenientes de Centroamérica, Panamá y México, así como investigaciones realizadas en países como Francia, Brasil, Alemania, Cuba, Holanda, Estados Unidos, entre otros.

Los países con la mayor parte de las publicaciones son Costa Rica y México 37 y 32\%, respectivamente (Figura 2). 


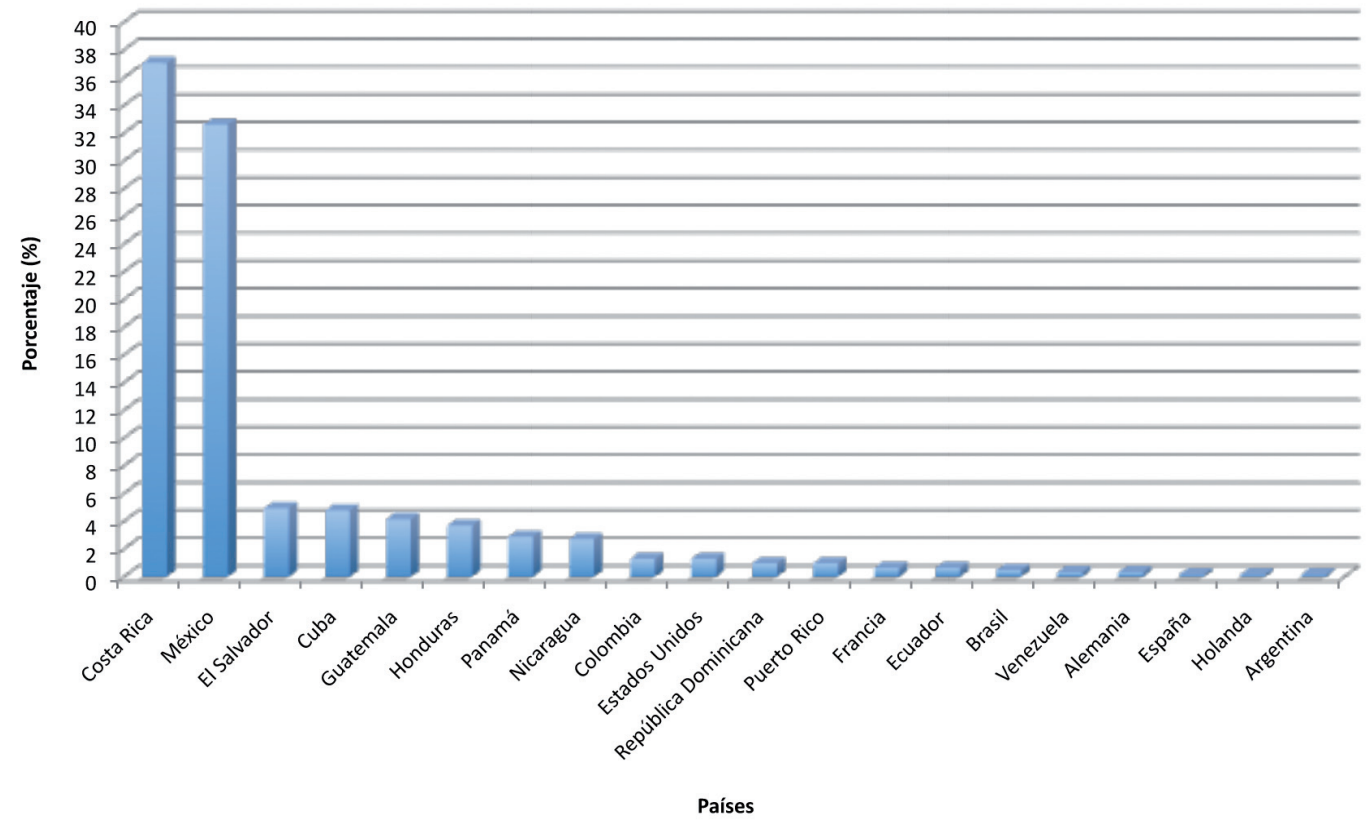

Figura 2. Porcentaje de trabajos publicados por país en la revista Agronomía Mesoamericana, durante el periodo 1990-2010. Costa Rica 2011.

\section{Temas relevantes}

En los últimos 21 años los temas han incluido todas las áreas agronómicas, y recientemente los relacionados con producción animal que han tomado gran auge. Algunas tópicos relevantes han incluido los granos básicos (maíz, frijol, arroz, sorgo), verduras y hortalizas (zanahoria, tomate, cebolla, espárragos, papas, lechuga, etc.), frutas (banano, papaya, fresa, piña, etc.) y productos de origen animal (leche, carne, pescado, etc.).

En maíz son más de 130 publicaciones (24\%) seguidas del frijol con 21\% (Figura 3). El 7\% de todas las publicaciones de la revista Agronomía Mesoamericana corresponden a temas que incluyen forrajes como la morera, el maní forrajero, la cratylia, el king grass, sorgo forrajero, etc. Otros tópicos relevantes de las investigaciones han sido el ganado lechero (4\%), el arroz, el sorgo, las malezas y el pejibaye (3\%).

Para ampliar la información sobre los temas evaluados, se puede observar la lista de trabajos publicada en éste volumen (Villegas 2011).

\section{Porcentaje de rechazo}

Con base en la información obtenida durante el año 2010, del total de trabajos recibidos, se rechazó un $20 \%$.

\section{Difusión impresa a nivel mundial}

Alrededor de treinta países forman parte de la lista de distribución; se incluyen principalmente los continentes de América, Europa, Asia y algunas de las islas del Caribe (Figura 4).

La revista países se envía a Estados Unidos, Francia, Holanda, Portugal, España, Italia, entre otros.

\section{AGRADECIMIENTOS}

Al Ing. Félix Zumbado Morales por haber iniciado la temática de este trabajo. A Meliza Villegas Alpízar y a Irene Ávalos Campos por su participación en la elaboración de este trabajo. 


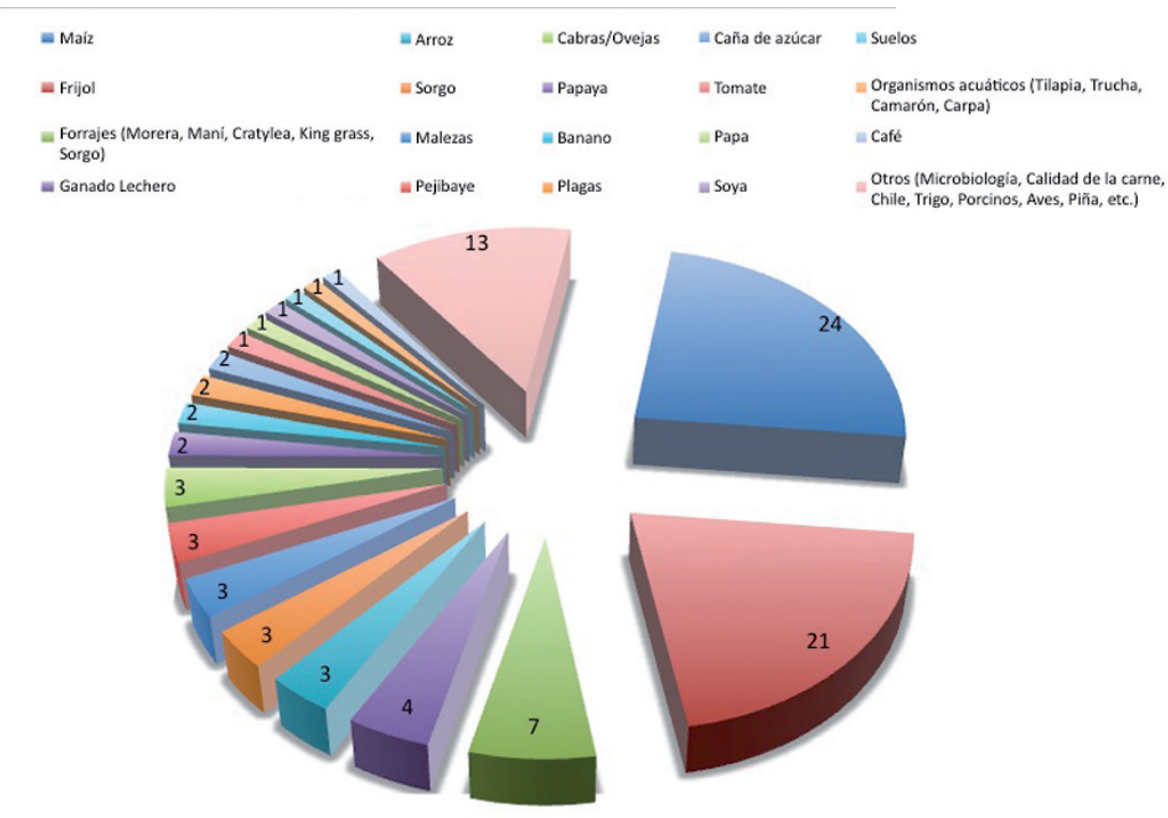

Porcentaje (\%)

Figura 3. Principales tópicos y la relación porcentual de las publicaciones realizadas en la revista Agronomía Mesoamericana durante el periodo comprendido entre 1990-2010. Costa Rica, 2011.

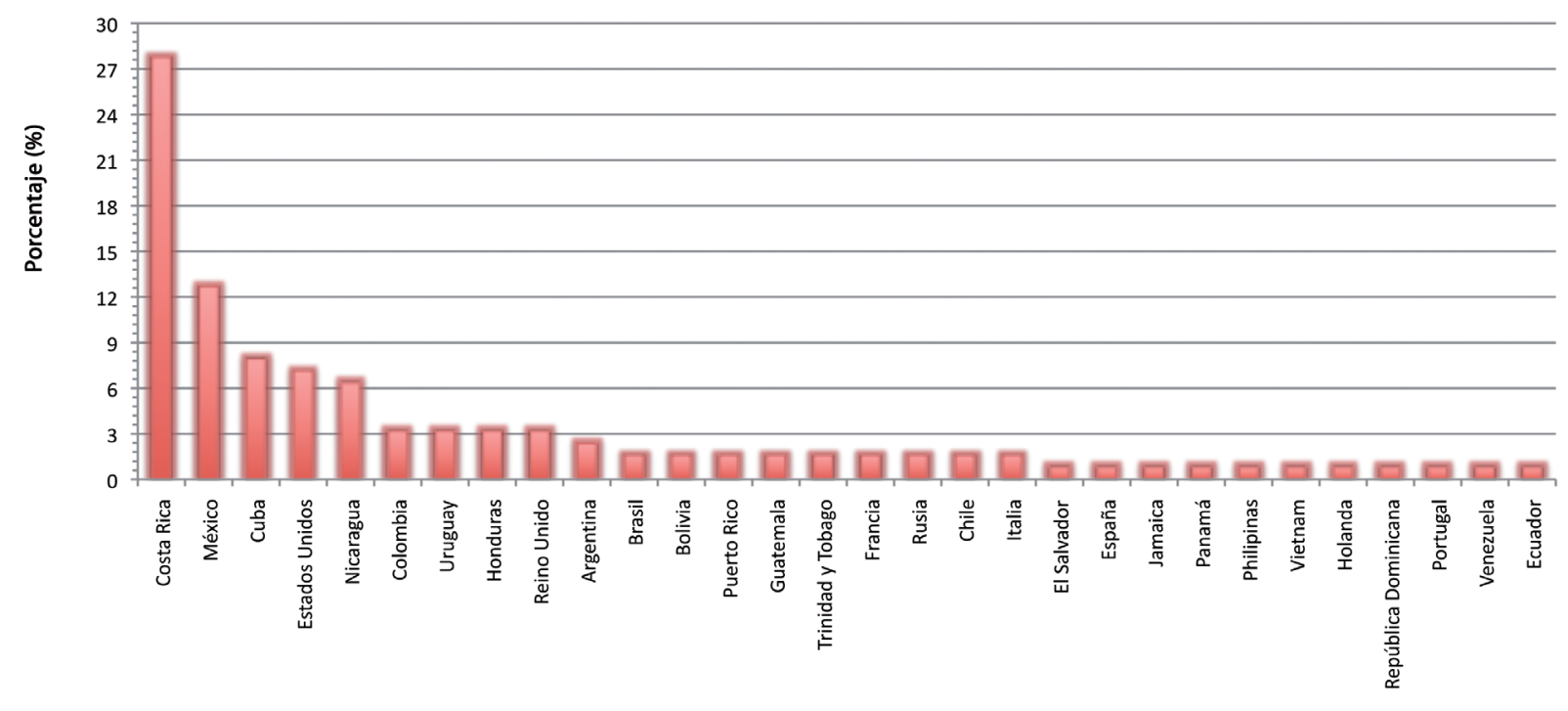

Países

Figura 4. Porcentaje de bibliotecas, centros de documentación, bases de datos por país, donde se envía la versión impresa de la revista Agronomía Mesoamericana a los diferentes países interesados en las investigaciones realizadas en la región Mesoamericana. Costa Rica, 2011. 


\section{LITERATURA CITADA}

Fuentes A; Salas C; Salazar A. 1990. Origen e historia del Programa Cooperativo Centroamericano y del Caribe para el Mejoramiento de Cultivos Alimenticios y Producción Animal. Agronomía Mesoamericana 1: 93-96.

Morera J; Astorga C. 1993. Reunión para la creación de la Red Mesoamericana de Recursos Fitogenéticos (REMERFI). Programa de Agricultura Sostenible. CATIE, Turrialba, Costa Rica. 129 p.

Villegas, M. 2011. Trabajos publicados en Agronomía Mesoamericana (1990-2010). Agronomía Mesoamericana 22(1):181-228.

Wellhausen E.J. 1990. Algunas reflexiones sobre el PCCMCA. Agronomía Mesoamericana 1:97-106.

\section{SITIOS DE INTERÉS}

Ministerio de Agricultura y Ganadería (MAG). Disponible en http://www.mag.go.cr/Agronomia_Mesoamericana/vol_anteriores.html

Estación Experimental Fabio Baurit. Disponible en http:// www.eefb.ucr.ac.cr/esp_ragromeso.shtml

Sistema Regional de Información para las Revistas Científicas de América Latina, el Caribe, España y Portugal (Latindex). Disponible en http://www.latindex.ucr. ac.cr/

Red de Revistas Científicas de América Latina y el Caribe, España y Portugal (Redalyc). Disponible en http://redalyc.uaemex.mx/src/inicio/HomRevRed. jsp?iCveEntRev=437 